\title{
Field analysis of two-dimensional focusing grating couplers
}

\author{
P.P. Borsboom* and H. J. Frankena \\ Research Group for Optics, Faculty of Applied Physics, Delft University of Technology, \\ Lorentzweg 1, 2628 CJ Delft, The Netherlands
}

Received July 29, 1994; revised manuscript received November 28, 1994; accepted January 5, 1995

The method that we have developed [P-P. Borsboom, Ph.D. dissertation (Delft University of Technology, Delft, The Netherlands); P-P. Borsboom and H. J. Frankena, J. Opt. Soc. Am. A 12, 1134-1141 (1995)] is successfully applied to a two-dimensional focusing grating coupler. The field in the focal region has been determined for symmetrical chirped gratings consisting of as many as 124 corrugations. The intensity distribution in the focal region agrees well with the approximate predictions of geometrical optics. In the case of short focusing grating couplers high-frequency intensity variations are observed in the focal region.

\section{INTRODUCTION}

A focusing grating coupler (FGC) redirects a guided wave propagating in a dielectric planar structure into a beam focused onto a point outside the waveguide, as discussed in several papers. ${ }^{1-3}$ In the future this device could become the replacement for the conventional lens utilized in compact disc players because it tends to be more compact, lighter, and more rigid than currently used optical systems. The diffraction of light by FGC's and their aberrations have been studied by geometrical-optics methods. ${ }^{1-3}$ The disadvantage of these methods is their inability to compute the actual field, or the intensity distribution, rigorously in the focal region. To overcome this limitation several methods treating two-dimensional gratings of finite length have been proposed, such as those of Liu and Chew ${ }^{4}$ and of Shigesawa and Tsuji. ${ }^{5}$ These methods could, in principle, be used to determine the field in the focal region. However, the former method considers only closed waveguides, whereas to our knowledge no literature has appeared describing the actual application of the latter method to FGC's. Furthermore, analysis of dielectric gratings of more than 20 periods has not been reported thus far, whereas at least 100 corrugations must be considered to represent a realistic FGC. Therefore we have developed a different method by which several two-dimensional dielectric optical gratings, comprising 100 or more corrugations, are treated in a numerically reliable way. ${ }^{6}$

In this method the grating is considered as a sequence of two alternating types of waveguide sections connected by means of step discontinuities, as shown in Fig. 1. The sections can vary in length, permitting the simulation of both periodic and nonperiodic (chirped) gratings. All $N$ waveguide layers are assumed to be homogeneous, isotropic, and linear. Losses in the media can be accounted for. All the fields are assumed to be time harmonic. The numerical examples presented here are confined to gratings comprising sequences of waveguide sections symmetric about the $x=0$ plane, although the proposed method can also be applied to asymmetrical profiles. In general, one can use symmetric gratings for qualitative considerations to gain a better understanding of grating behavior.

\section{PROPOSED METHOD OF ANALYSIS}

To obtain the field scattered by the entire grating we begin by determining the field scattered by a single step. The scattering properties of this step are expressed by a scattering matrix. Combining the matrices of the various steps and waveguide sections, we obtain a matrix describing the entire grating.

The approach followed can be divided into three stages:

A. Modal representation of the fields present in the planar waveguide sections,

B. Scattering characteristics of a step discontinuity,

C. Combination of the scattering properties of a large number of alternating step discontinuities and planar waveguide sections.

All three stages were treated in Refs. 6 and 7 and are only briefly discussed here.

We introduce a right-handed Cartesian system of coordinates, as indicated in Fig. 1, where $x$ is the direction perpendicular to the interfaces between the homogeneous dielectric layers and $z$ is the propagation direction of the guided waves. All the field quantities and the geometry are assumed to be independent of $y$. Using the modal representation in terms of guided and radiation modes, as discussed, e.g., by Marcuse ${ }^{8}$ and Vassallo, ${ }^{9}$ we can express the fields by

$$
\begin{aligned}
E_{y}(x, z)= & \sum_{m=0}^{M-1}\left[A_{m} \exp \left(-j \kappa_{m} z\right)+B_{m} \exp \left(j \kappa_{m} z\right)\right] \psi_{m}(x) \\
& -\int_{0}^{\infty} \frac{k_{x 1}}{k_{z 1}}\left[a_{1}\left(k_{x 1}\right) \exp \left(-j k_{z 1} z\right)\right. \\
& \left.+b_{1}\left(k_{x 1}\right) \exp \left(j k_{z 1} z\right)\right] \psi_{A}\left(k_{x 1}, x\right) \mathrm{d} k_{x 1} \\
& -\int_{0}^{\infty} \frac{k_{x N}}{k_{z N}}\left[a_{N}\left(k_{x N}\right) \exp \left(-j k_{z N} z\right)\right. \\
& \left.+b_{N}\left(k_{x N}\right) \exp \left(j k_{z N} z\right)\right] \psi_{A}\left(k_{x N}, x\right) \mathrm{d} k_{x N}
\end{aligned}
$$

where $\psi_{m}, \psi_{A}$, and $\psi_{B}$ are wave functions, as described in 


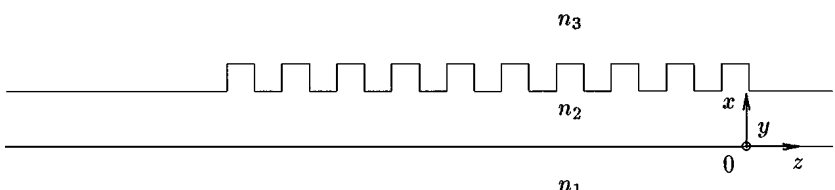

Fig. 1. Relief-type grating.

Refs. 6 and 7. The subscript $N$ in $k_{x N}$ and $k_{z N}$ indicates that these quantities are considered in the superstratum. $M$ denotes the number of guided modes that are confined to the guiding layer of the waveguide. Furthermore, $A_{m}$, $a_{1}\left(k_{x 1}\right)$, and $a_{N}\left(k_{x N}\right)$ are the complex amplitude coefficients of the guided and the radiation modes that propagate in the positive $z$ direction, whereas $B_{m}, b_{1}\left(k_{x 1}\right)$, and $b_{N}\left(k_{x N}\right)$ are the corresponding quantities for the modes traveling in the negative $z$ direction. $\quad k_{z 1}$ and $k_{z N}$ are the propagation constants of the radiation modes $\psi_{A}$ and $\psi_{B}$, respectively. They are defined as $k_{z 1}=\left(n_{1}^{2} k_{0}^{2}-k_{x 1}{ }^{2}\right)^{1 / 2}$ and $k_{z N}=\left(n_{N}^{2} k_{0}^{2}-k_{x N}^{2}\right)^{1 / 2}$. Here $n_{1}$ and $n_{N}$ are the complex refractive indices of the substrate and the superstratum, respectively, and $k_{0}=2 \pi / \lambda_{0}$, where $\lambda_{0}$ is the free-space wavelength. Finally, the modal expansion of $H_{x}$ can be obtained from Eq. (1) by application of the relationship $H_{x}=[j \omega \mu(x)]^{-1} \partial E_{y} / \partial z$.

Note that, because the configuration treated in the numerical examples is symmetric with respect to $x$, we have introduced a different set of radiation modes $\psi_{E}$ and $\psi_{O}$ that are linear combinations of $\psi_{A}$ and $\psi_{B}$ :

$$
\begin{aligned}
& \psi_{E}=\psi_{A}+\psi_{B}, \\
& \psi_{O}=\psi_{A}-\psi_{B},
\end{aligned}
$$

where $\psi_{E}$ is even with respect to the plane $x=0$ and $\psi_{O}$ is odd. The superior numerical characteristics of the modal expansion that we obtain by choosing these latter modes were demonstrated by Vassallo. ${ }^{10}$ In all the examples presented below, the field incident upon the FGC will be a $\mathrm{TE}_{0}$ guided mode (which is an even function of $x$ ); therefore only the $a_{1}$ and the $b_{1}$ coefficients must be considered in these examples. The $\mathrm{TE}_{0}$ mode is defined as

$\psi_{0}(x)=\left\{\begin{array}{l}A_{0, N} \exp \left[-j k_{x N}\left(x-X_{N-1}\right)\right] \\ B_{0, p} \exp \left[-j k_{x p}\left(x-X_{p}\right)\right]+C_{0, p} \exp \left[+j k_{x p}\left(x-X_{p}\right)\right] \\ D_{0,1} \exp \left[+j k_{x 1}\left(x-X_{1}\right)\right]\end{array}\right.$

where $A_{0, N}, B_{0, p}$, and $C_{0, p}$ can be expressed in terms of $D_{0,1}$ through the boundary conditions at the interfaces $X_{p}(p=1, \ldots, N-1)$ between the $N$ homogeneous waveguide layers.

To determine the field scattered by a single step discontinuity, the mode-matching method ${ }^{11-14}$ is applied. To this aim, the modal expansion (1), with unknown amplitude coefficients, is used to represent the tangential field components $E_{y}$ and $H_{x}$ at both sides of the step. These field components are then forced to be continuous across the plane of the step, and the resulting equations are solved to yield the unknown amplitude coefficients. In this process the integrals representing the radiation fields are truncated, and the remaining integration intervals are divided into subintervals. In each subinterval the amplitude coefficients are expanded by use of Chebyshev polynomials. By this method the field on both sides of the step discontinuity is represented by a set of expansion coefficients, which are arranged as a column matrix to comply with the scattering matrix formalism. This formalism was chosen because of its well-known numerical stability.

We thus obtain

$$
\left(\begin{array}{l}
W_{2}^{+} \\
W_{1}^{-}
\end{array}\right)=\left(\begin{array}{ll}
S_{11} & S_{12} \\
S_{21} & S_{22}
\end{array}\right)\left(\begin{array}{l}
W_{1}^{+} \\
W_{2}^{-}
\end{array}\right),
$$

where the square matrix is called the scattering matrix pertaining to the step discontinuity at $z=z_{0}$. Here $W_{i}^{+}=\left[A_{m}^{(i)}, a_{1, r, s}^{(i)}, a_{N, r, s}^{(i)}\right]^{T}(i=1,2)$ are column matrices that describe the waves traveling in the positive $z$ direction and $W_{i}^{-}=\left[B_{m}^{(i)}, b_{1, r, s}^{(i)}, b_{N, r, s}^{(i)}\right]^{T}(i=1,2)$ are column matrices that describe waves traveling in the negative $z$ direction, at the left- and the right-hand sides of the step discontinuity depending on $i=1$ or $i=2$, respectively. $\quad a_{1, r, s}^{(i)}$ and $a_{N, r, s}^{(i)}$ are the coefficients of the $r$ th-order Chebyshev polynomial used in the expansion of $a_{1}$ and $a_{N}$ in the $s$ th interval of the truncated integration domain. Similarly, $b_{1, r, s}^{(i)}$ and $b_{N, r, s}^{(i)}$ correspond to the expansions of $b_{1}$ and $b_{N}$, respectively. The scattering matrix corresponding to the planar waveguide section bounded by the planes at $z=z_{k}$ and at $z=z_{k+1}$ is defined as

$$
\left(\begin{array}{c}
W_{k+1}^{+} \\
W_{k}{ }^{-}
\end{array}\right)=\left(\begin{array}{ll}
P & 0 \\
0 & P
\end{array}\right)\left(\begin{array}{c}
W_{k}^{+} \\
W_{k+1}{ }^{-}
\end{array}\right),
$$

where the matrix $P$ relates the complex amplitudes of the waves traveling in both the positive and the negative $z$ direction. Here $W_{k}$ and $W_{k+1}$ denote the wave amplitudes traveling to the left and the right, respectively, at $z=z_{k}$ and at $z=z_{k+1}$. In combining these matrices into a matrix describing the entire grating, the Redheffer product has been applied. ${ }^{15}$

All the above steps were implemented by a computer program that can be used to determine the field scattered by periodic or aperiodic gratings. Periodic gratings were successfully treated in our previous paper. ${ }^{6}$ There-

$$
\begin{aligned}
& \text { for } X_{p-1}<x<X_{p} \text {, } \\
& \text { for } x<X_{1}
\end{aligned}
$$

fore only the focal-region field of chirped gratings is discussed here.

\section{NUMERICAL RESULTS}

In this section we discuss a symmetrical chirped grating, which is the two-dimensional equivalent of the FGC. The $z$ coordinates of the scattering steps were determined by geometrical optics, according to Ref. 16, and the focalregion field scattered by the grating was computed. The configuration of the chirped grating closely resembles the periodic grating discussed in our previous paper. ${ }^{6}$ This periodic grating was derived from the examples presented in the papers by Liu and Chew ${ }^{4}$ and by Shigesawa and Tsuji. ${ }^{5}$ For the chirped grating the distances between 


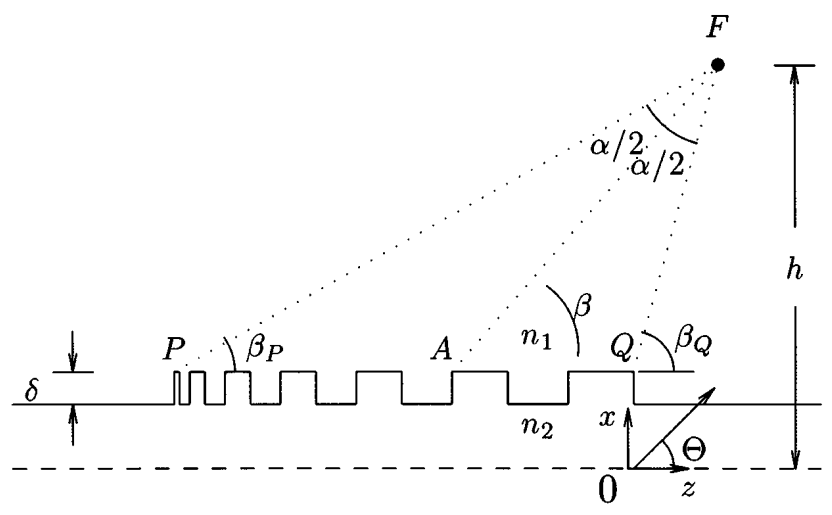

Fig. 2. Geometry of the grating.

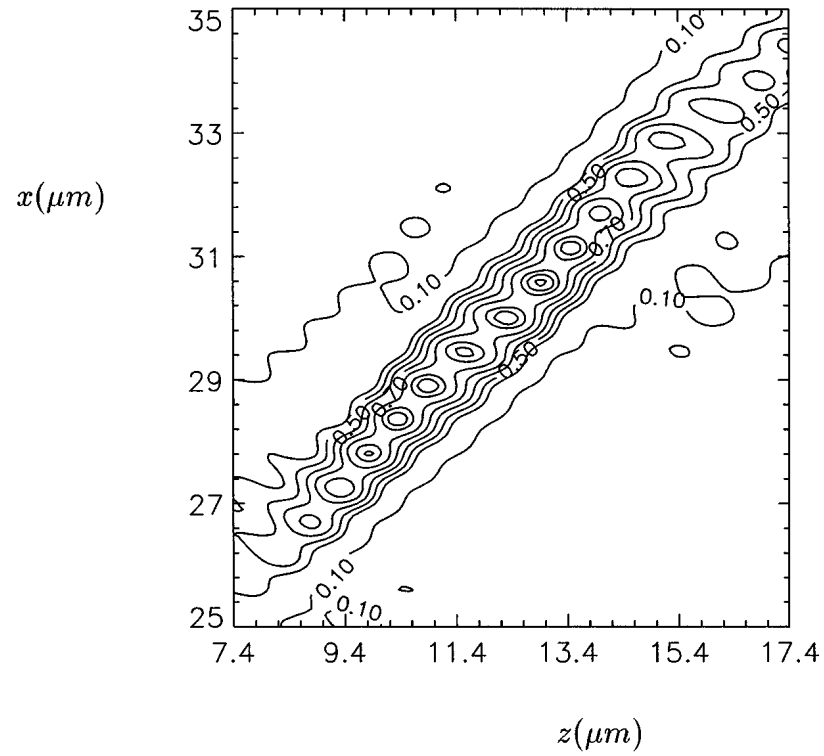

Fig. 3. Focal region of a 19-corrugation grating with $\beta=45^{\circ}$, $h=30 \mu \mathrm{m}$.

adjacent steps increase with $z$, and it is more appropriate to replace the word period with the word corrugation.

Using the method described in Section 2, we designed three chirped gratings in three-layer waveguide structures, each with the geometry indicated in Fig. 2, in which only the upper half $(x>0)$ of a symmetrical chirped grating is shown. The gratings are $2 h$ long and have their focal points at $(h, 0.414 h)$. This geometry was chosen such that the angle $\alpha$ between $P F$ and $Q F$ is $45^{\circ}$, whereas the angle $\beta$ between $A F$ and the $x$ axis equals $45^{\circ}$. Here $\beta$ is the average angle under which the grating radiates toward its focus. Furthermore, when we define the numerical aperture NA as the sine of $\alpha / 2$, for these configurations $\mathrm{NA}=0.38$. Calculations were performed for $h=30 \mu \mathrm{m}, h=80 \mu \mathrm{m}$, and $h=200 \mu \mathrm{m}$, respectively. In all three geometries $\delta=0.08 \mu \mathrm{m}$.

The positions of the steps in these gratings were chosen such that, if a Gaussian light beam travels from the beginning of the grating at $z=-2 h$ through a step (whose height is neglected in this approximation) toward the focal point, its optical path equals a constant plus $l \lambda_{0}$, where $l=0, \pm 1, \pm 2, \ldots$. Hence all the scattered contributions arrive at the focal point in phase, producing a maximum intensity there. The positions of the steps are then given $\mathrm{by}^{16}$ :

$$
\begin{aligned}
z_{N_{s}}= & \frac{n_{g} n_{f} h}{n_{g}{ }^{2}-n_{f}{ }^{2}} \\
& \times\left[1-\frac{N_{s} \lambda_{0}}{2 n_{f} h}-\left(1-\frac{n_{f} N_{s} \lambda_{0}}{n_{g}{ }^{2} h}+\frac{N_{s}{ }^{2} \lambda_{0}{ }^{2}}{4 n_{g}{ }^{2} h^{2}}\right)^{1 / 2}\right],
\end{aligned}
$$

where $z_{N_{s}}$ denotes the $z$ coordinate of the $N_{s}$ th step, $n_{f}$ is the refractive index of the medium in which the focus is situated, and $n_{g}$ is the average of the two effective indices corresponding to the two waveguides making up the grating.

In Fig. 3 a computed plot of the focal-region intensity of a 19-corrugation grating with $h=30 \mu \mathrm{m}$, with its geometrical-optics focal point at $(30,12.42 \mu \mathrm{m})$, is shown. The focal-region intensity distribution is roughly elliptical, with its main axis making an angle of approximately $45^{\circ}$ with the $x$ axis, which is in agreement with the geometrical-optics considerations presented above. The length of the area in which the field intensity is equal to, or more than, one half of the maximum intensity in focus is approximately $9 \mu \mathrm{m}$. The width, where the field intensity is 0.8 of the maximum intensity, is approximately $1.8 \mu \mathrm{m}$. This result agrees well with estimates of the dimensions ( 8 and $2 \mu \mathrm{m}$, respectively) and of the orientation of the focus that we obtained by considering the grating as an aperture and subsequently applying geometrical-optics methods and scalar diffraction theory. ${ }^{17}$

A ripple in the focal-region intensity distribution can be observed in Fig. 3. Through convergence checks we established that the ripple is not caused by numerical noise. All the experimental work and most of the approximate ray-tracing methods deal only with long gratings (1000 periods or more). By changing only $h$ in the configuration of Fig. 2 and observing the calculated field in the focal region for gratings of $2 h=160 \mu \mathrm{m}$ (50 corrugations) and $2 h=400 \mu \mathrm{m}$ (124 corrugations), we can see that the ripple becomes less pronounced, as shown in Figs. 4 and 5. This effect suggests its absence in the fields of longer gratings. However, further computations will have to be carried out to confirm this result and to explain the occurrence of the short gratings' ripples.

For the 19-corrugation grating, the complex amplitude coefficients are shown in Fig. 6 for the reflected radiation field at $z<-2 h$ and in Fig. 7 for the transmitted field at $z>0$. We can see from Eq. (1) that the coefficients $a_{1}$ and $b_{1}$ appear only in combination with the factor $k_{x 1} / k_{z 1}$. Therefore, to assess the actual contributions of these coefficients on the intensity, this factor must be taken into account because it has a broadening effect along the $k_{x 1}$ axis. We can observe from the plots that the transmitted field consists mainly of radiation modes with $k_{x 1}$ values in the $1.0 \mu \mathrm{m}^{-1}<k_{x 1}<3.5 \mu \mathrm{m}^{-1}$ range. Note that this $k_{x 1}$ domain is roughly predictable from the grating configuration. By assuming that the wave vector $\mathbf{k}$ in the superstratum corresponds to a radiation angle $\Theta$, this angle can be computed from the modulus of the wave vector $|\mathbf{k}|=$ $n_{1} k_{0}$ and from its $x$ component $k_{x 1}$ with the equation $\Theta=$ $\sin ^{-1}\left(k_{x 1} /|\mathbf{k}|\right)$. From the two angles $\beta_{P}$ and $\beta_{Q}$ shown in Fig. 2 we can determine the boundaries of the $k_{x 1}$ inter- 


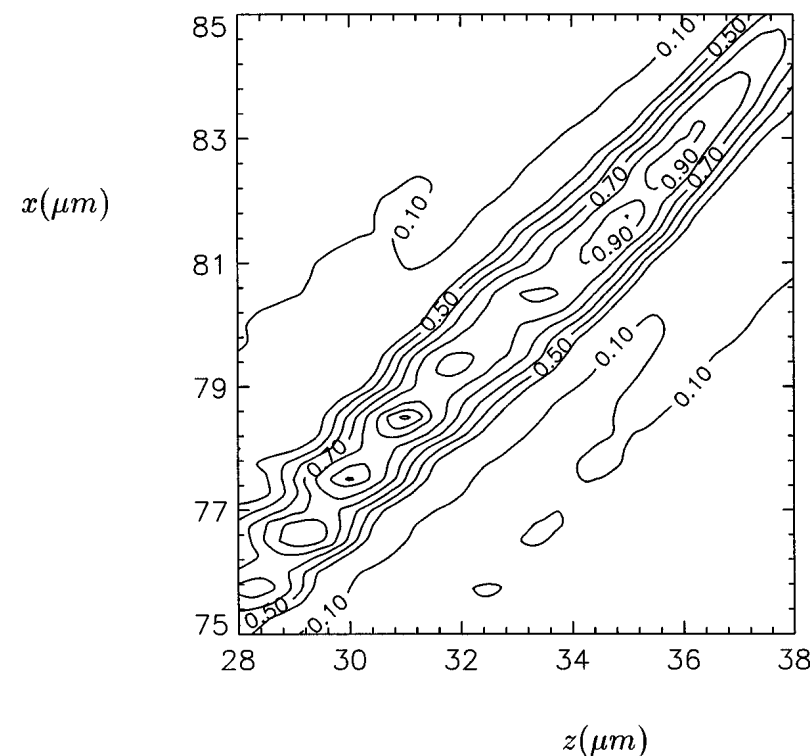

Fig. 4. Focal region of a 50-corrugation grating with $\beta=45^{\circ}$, $h=80 \mu \mathrm{m}$.

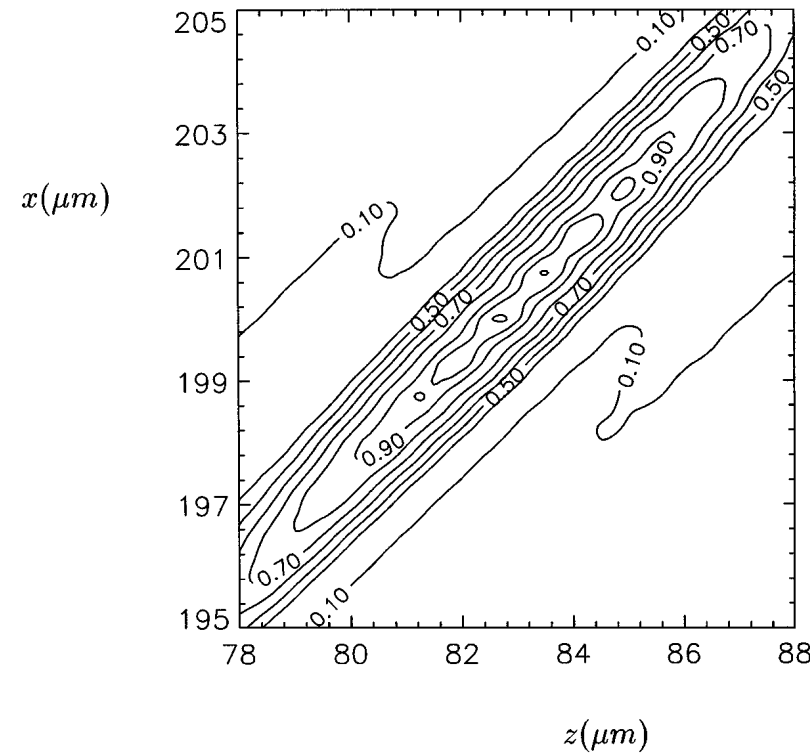

Fig. 5. Focal region of a 124-corrugation grating with $\beta=45^{\circ}$, $h=200 \mu \mathrm{m}$.

val responsible for the dominant contribution to the radiation field, as described in Ref. 6. This determination in turn enables us to approximate the amplitude coefficients efficiently because only this interval must be sampled accurately, i.e., must be divided into many subintervals (typically 100).

We can observe from Fig. 7 that the maxima of the real and imaginary parts of amplitude $a_{1}$ lie at $k_{x 1} \approx 2$, corresponding to an angle $\Theta \approx 30^{\circ}$, which is different from that predicted by geometrical optics $\left(\Theta=45^{\circ}\right)$. This result may be explained by the fact that the amplitude of the guided wave decreases as it propagates through the grating, whereas in the geometrical-optics analysis this phenomenon is neglected. However, this is just one possible explanation, and further investigation is required for a full understanding of this discrepancy.
Comparison of the complex amplitudes $a_{1}$ and $b_{1}$ with those of a periodic grating ${ }^{6}$ shows that the $a_{1}$ and the $b_{1}$ values of a periodic grating have a peaked character, whereas those corresponding to a chirped grating are more evenly distributed over the interval $0<k_{x 1}<n_{1} k_{0}$, although oscillations occur. This is because for a periodic grating the radiation occurs predominantly in a single direction, whereas a chirped grating scatters the light in several directions, thereby creating a focusing effect. In the case of periodic gratings, we observed from numerical experiments that, especially for longer gratings, the amplitude coefficient is reduced almost to a single narrow peak. Therefore we can, in most cases, restrict the integrations of Eq. (1) to a subinterval near this peak, thereby greatly increasing the efficiency of the computation. By comparison, the chirped grating has an amplitude coefficient whose width is dependent only on the NA of the grating, not on the number of corrugations. However, all things considered, our experience is that the computation of the amplitude coefficients corresponding to either a periodic or a chirped grating requires approximately the same computation effort for an identical number of steps, even though the coefficients involved are entirely different.

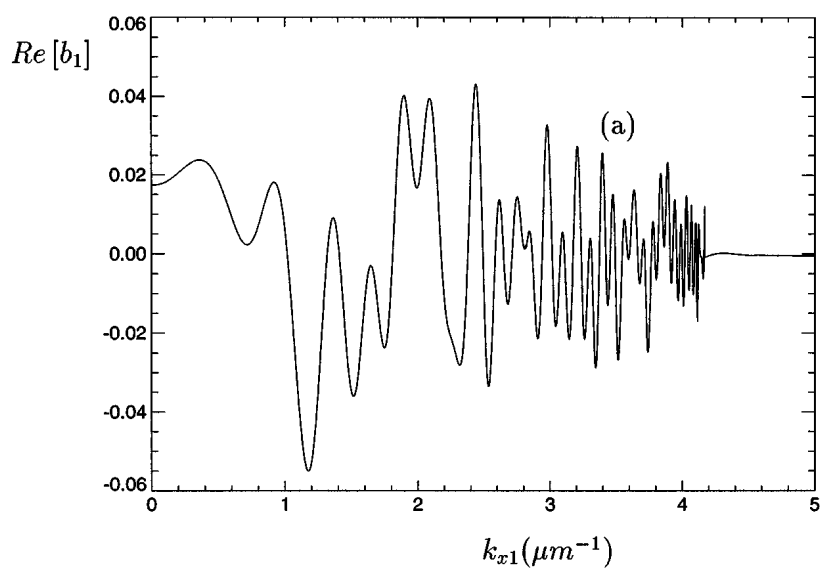

(a)

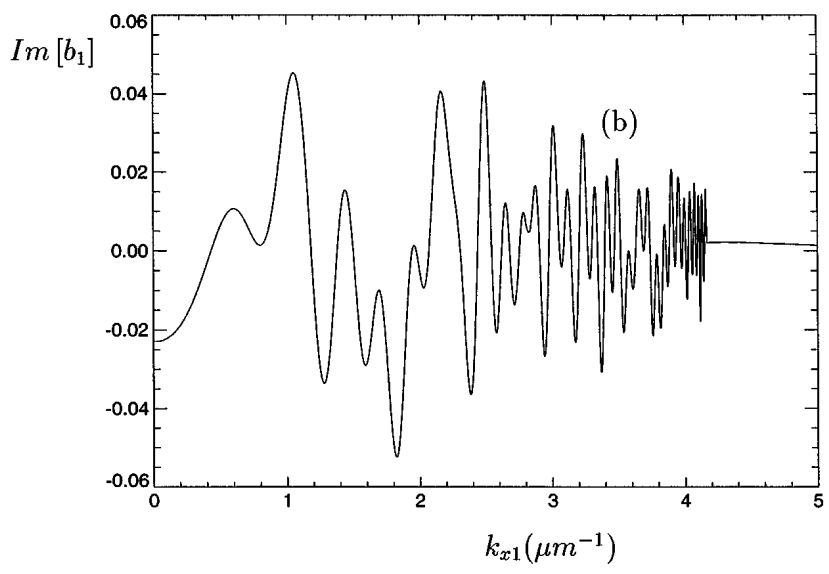

(b)

Fig. 6. Amplitude coefficient $b_{1}(\mathrm{~V})$ corresponding to the reflected radiation field of the FGC comprising 19 corrugations: (a) real part, (b) imaginary part. 


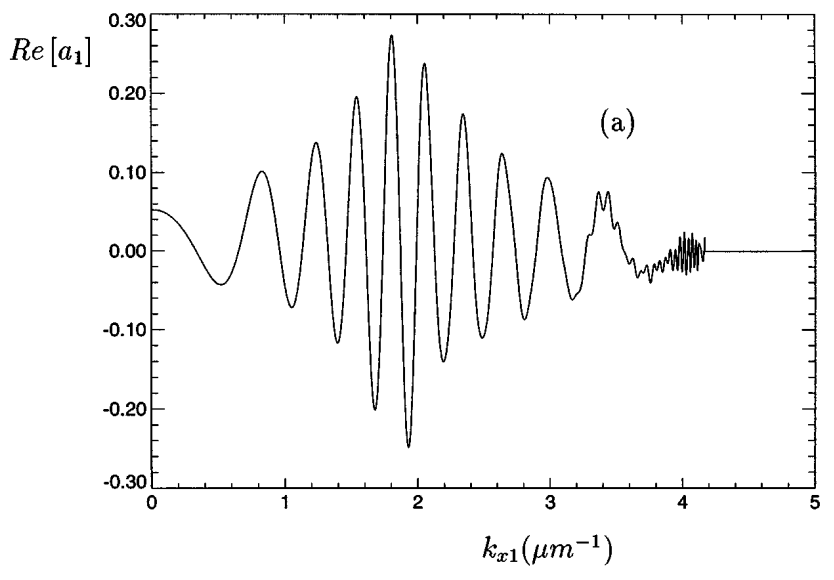

(a)

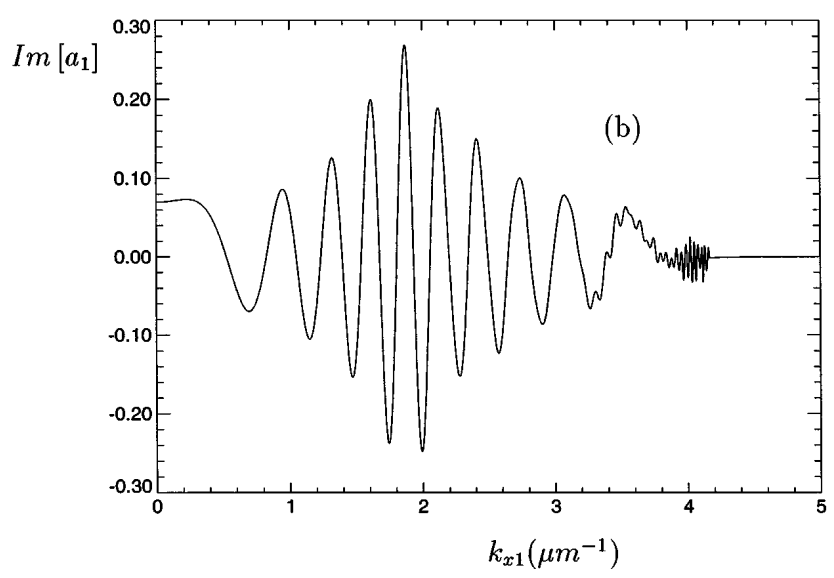

(b)

Fig. 7. Amplitude coefficient $a_{1}$ (V) corresponding to the transmitted radiation field of the FGC comprising 19 corrugations: (a) real part, (b) imaginary part.

\section{CONCLUSIONS}

In this paper the method presented in Refs. 6 and 7 has been successfully applied to analyze the field produced by a two-dimensional focusing grating coupler. Focalregion fields have been determined for three symmetrical gratings with 19,50 , and 124 corrugations. The intensity distribution in the focal region agrees well with the approximate predictions of geometrical optics. In the case of a focusing grating coupler of limited length, high-frequency intensity variations were observed in the focal region. The amplitude of these variations is lowered when the length of the grating increases. A plausible explanation of this phenomenon has not yet been found and is the object of ongoing research.

\section{ACKNOWLEDGMENTS}

The financial support of Philips Research Laboratories is gratefully acknowledged. We thank J. J. M. Braat of
Philips Research Laboratories and of the Delft University of Technology for his contributions to the research described in this paper.

*P-P. Borsboom is now with the Rutherford Appleton Laboratory, Chilton, Didcot, Oxfordshire OX11 0QX, UK.

\section{REFERENCES}

1. S. Ura, T. Suhara, H. Nishihara, and J. Koyama, "Focusing grating for integrated optical disc pickup device," Trans. Inst. Electron. Commun. Eng. Jpn. Part C (Japan) 68-C, 803-811 (1985).

2. S. Ura, T. Suhura, and H. Nishihara, "Aberration characterizations of a focusing grating coupler in an integrated-optic disc pickup device,” Appl. Opt. 26, 4777-4782 (1987).

3. J. J. M. Braat and M. O. E. Laurijs, "Geometrical optics design and aberration analysis of a focusing grating coupler," Opt. Eng. 33, 1037-1043 (1994).

4. Q.-H. Liu and W. C. Chew, "Analysis of discontinuities in planar dielectric waveguides: an eigenmode propagation method," IEEE Trans. Microwave Theory Tech. 39, 422-430 (1991).

5. H. Shigesawa and M. Tsuji, "A new equivalent network method for analyzing discontinuity properties of open dielectric waveguides," IEEE Trans. Microwave Theory Tech. 37, 3-14 (1989).

6. P-P. Borsboom and H. J. Frankena, "Field analysis of twodimensional integrated optical gratings," J. Opt. Soc. Am. A 12, 1134-1141 (1995).

7. P-P. Borsboom, "Field analysis of two-dimensional integrated-optical gratings," Ph.D. dissertation (Delft University of Technology, Delft, The Netherlands, 1994).

8. D. Marcuse, Theory of Dielectric Optical Waveguides (Academic, New York, 1974), Chap. 1, pp. 1-30.

9. C. Vassallo, Théorie des Guides D'Ondes Électromagnétiques (Eyrolles, Paris, 1985), Chap. 3, pp. 151-248.

10. C. Vassallo, "Radiating normal modes of lossy planar waveguides," J. Opt. Soc. Am. 69, 311-316 (1979).

11. S. F. Mahmoud and J. C. Beal, "Scattering of surface waves at a dielectric discontinuity on a planar waveguide," IEEE Trans. Microwave Theory Tech. MTT-23, 193-198 (1975).

12. W. Biehlig, K. Hehl, U. Langbein, and F. Lederer, "Light propagation in a planar dielectric slab waveguide with step discontinuities. Part I. Operator formalism," Opt. Quantum Electron. 18, 219-228 (1986).

13. W. Biehlig, "Light propagation in a planar dielectric slab waveguide with step discontinuities. Part 2. Numerical analysis of TE-polarized fields," Opt. Quantum Electron. 18, 229-238 (1986).

14. H. Shigesawa and M. Tsuji, "Mode propagation through a step discontinuity planar waveguide," IEEE Trans. Microwave Theory Tech. MTT-34, 205-212 (1986).

15. R. Redheffer, "Difference equations and functional equations in transmission-line theory," in Modern Mathematics for the Engineer, 2nd ed., E. F. Beckenbach, ed. University of California Engineering Extension Series (McGraw-Hill, New York, 1961), pp. 282-337.

16. J. J. M. Braat, "Microscope objectives for optical disc systems," in Huygens' Principle 1690-1990: Theory and Applications, H. Blok, H. A. Ferwerda, and H. K. Kuiken (Elsevier Science B. V., Amsterdam, 1990), pp. 33-63.

17. M. Born and E. Wolf, Principles of Optics (Pergamon, New York, 1980), Chap. 8, pp. 439-441. 